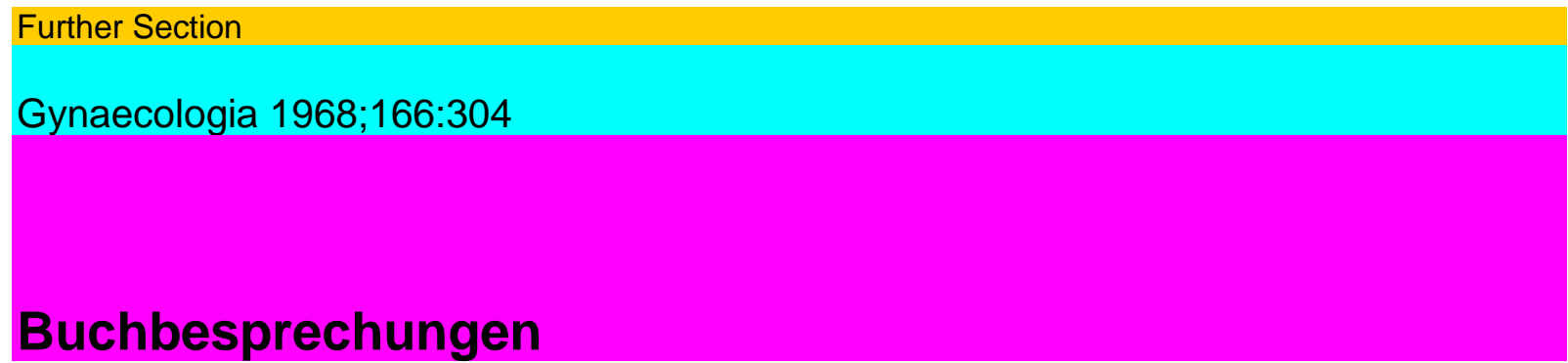

\title{
BOOK REVIEWS LIVRES NOUVEAUX
}

Jeffcoate, T. N. A.: Principles of Gynecology. Butterworth, London 1967. 971 p. Preis: E6 s. 10. In diesem Werk werden neben der allgemeinen Gynäkologie auch die Gebiete derEndokrinologie, Genetik, Biochemie, Psychologie, Präventivmedizin und Familien-planung ausführlich behandelt. Atiologie, klinische Symptomatik, Diagnostik sowiekonservative und operative Therapie werden gleichermassen gründlich besprochen.Dieser ganze Theinenkreis wird durchwegs vom Standpunkt des klinisch tätigenFrauenarztes aus betrachtet. Dank seiner Struktur dürfte dieses Buch weniger alsLehrbuch für Studierende, sondern viel eher als kleines Handbuch für in der Aus-bildung oder in der Praxis stehende Gynäkologen geeignet sein. H. Erb, Bern

Breit, A.: Angiographie der Uterustumoren und ihrer Rezidive. Thieme, Stuttgart 1967. Preis: DM 39,-.

Der Titel der vorliegenden Monographie ist etwas irreführend, da es sich aus-schliesslich um Arteriographien handelt und zudem vorwiegend das Material von Angiographien am Kollumkarzinom gesammelt wurde. Nach der Schilderung der normalen anatomischen Verhältnisse werden die Befunde bei Kollumkarzinom gezeigt, wobei im wesentlichen zwei für die Therapie wichtige Kriterien differenziert werden können. Das erste ist der Gefässreichtum des Tumors, von dem seine Strah-lensensibilität abhängig ist; das zweite Kriterium ist die Tiefe der parametranen Infiltration, die arteriographisch Differenzialdiagnosen zwischen wahrer Infiltration und entzündlichen Vorgängen erlaubt.

Es ist nicht vorauszusehea, ob die Arteriographie zukünftig für den Onkologendie gleiche Bedeutung erreichen wird, wie es die Lymphographie heute schon hat.Sollte dies der Fall sein, so wird dieses Buch dank seines vorzüglichen Bildmaterialsden Ausschlag hierzu geben. W. Obolensky, Liestal

Ludwig, H.: Míkrozirkulationsstörungen und Diapedeseblutungen im fetalen Gehirn bei Hypoxie. Bibliotheca Gynaecologica No. 46. Karger, Basel/New York 1968. VIII + 168 p., 105 fig., 10 tab. Preis: sFr./DM 46.-, US\$ 11.05/84s.

D. Der Autor weist in sehr schön dokumentierten Tierversuchen nach dass diedurch Anoxämie induzierten Gehirnblutungen mit einer erhöhten Fibrinolyseaktivi-tät einhergehen. Im weiteren konnte der Verfasser zeigen dass durch die Zuführungvon Fibrinolyseinhibitoren an das Muttertier eine Verminderung dieser Gehirnblutungen möglich ist. Der vom Autor angedeutete neue Weg einer Prophyïaxe perinataler Schockfolgen durch die Verabreichung von Fibrinolyseinhibitoren an dieMutter stellt die Behandlung eines Symptoms und nicht der zugrundeliegendenAnoxämie und Azidose dar und dürfte sich deshalb nicht durchsetzen. Das Buch istvon besonderem Interesse für Perinatologen geburtshiliicher und pädiatrischerRichtung sowie für Neurologen. W. Obolensky Liestal 